\title{
Guillain-Barre syndrome as the first manifestation of juvenile systemic lupus erythematosus: a case report
}

This article was published in the following Dove Press journal:

Open Access Rheumatology: Research and Reviews

\section{Vadood Javadi Parvaneh \\ Mohsen Jari \\ Saeideh Qhasemi \\ Mohammad Mahdi Nasehi \\ Khosro Rahmani \\ Reza Shiari}

Mofid Children's Hospital, Shahid Beheshti Medical University of Sciences, Tehran, Iran
Correspondence: Reza Shiari Mofid Children's Hospital, Higher than Hosseinieh Ershad, Shariati Avenue,

Tehran, Iran

Email shiareza@yahoo.com
Background: Systemic lupus erythematosus (SLE) is an autoimmune disease which involves multiple organs, including peripheral nervous system.

Case presentation: We describe a 12-year-old boy with progressively worsening neurological symptoms as first manifestation. Legs pain, loss of balance, and lower extremity weakness were the reason for his admission in neurologic ward. The patient was started on intravenous immunoglobulin therapy due to the possibility of GuillainBarre syndrome and acute inflammatory demyelinating polyneuropathy (AIDP). However, there was no appropriate response and he developed recurrent attacks of polyneuropathy again with diagnosis of chronic inflammatory demyelinating polyneuropathy (CIDP). Then, he received intravenous pulse of methylprednisolone for 5 consecutive days followed by oral prednisolone for 3 months. One month after withdrawal of corticosteroid he admitted again with the same manifestations. Rheumatologic workup revealed the presence of leukopenia, hemolytic anemia, hematuria, proteinuria, positive antinuclear antibodies, and ds-DNA antibodies. On the basis of the American College of Rheumatology and Systemic Lupus International Collaborating Clinics Classification Criteria for SLE, the patient had underlying diagnosis of SLE. Eventually, he was treated by the pulse of methylprednisolone and cyclophosphamide, and oral hydroxychloroquine and prednisolone. His neurological and physical symptoms improved and complete neurological recovery occurred several months later.

Conclusion: SLE and AIDP/CIDP are different entities, but ADP/CIDP can be part of the neurologic manifestations of the SLE. Although the association between AIDP/CIDP and SLE is very rare especially as a first manifestation of SLE, it should be early recognized for rapid appropriate treatment.

Keywords: Guillain-Barre syndrome, chronic inflammatory demyelinating polyneuropathy, systemic lupus erythematosus, nerve disorders

\section{Introduction}

Systemic lupus erythematosus (SLE) is an autoimmune systemic disorder with high mortality rate. The exact cause of this disease is unknown; however, several factors such as genetics, environment, drugs, pregnancy, and infection are involved in SLE. ${ }^{1}$ This disease adversely affects multiple organs such as mucocutaneous, joints, kidneys, lungs, hearts and particularly nerve cells in the peripheral nervous system. ${ }^{2}$ Guillain-Barre syndrome (GBS) is a rare autoimmune disease which attacks the nervous system. ${ }^{3}$ Recent studies have demonstrated that SLE may be rarely 
associated with GBS; however, the exact mechanism of it is unclear. To our knowledge, a few cases of juvenile SLE at different ages have been reported with the initial manifestation of GBS. ${ }^{2,4}$ Herein, we reported a 12-year-old boy with SLE who initially presented with GBS.

\section{Case presentation}

A 12-year-old boy with previous history of upper respiratory tract infection was referred to outpatient clinic of Mofid Children Hospital because of legs pain, walking instability, and lower extremity weakness. His problems were started since 5 days ago. The past medical history revealed that he was a third child of the family (III/III). There was a familiar relationship between his parents (cousin relationship). Furthermore, his 22-year-old sister had a history of lupus. His mother had also the history of three abortions. On physical examinations, he had decreased in deep tendon reflexes (DTR). His first biochemical tests results were as following: white blood count $=3,650 / \mathrm{mm}^{3}$ (neutrophil: $44 \%$ and lymphocyte: $56 \%$ ); hemoglobin $=13 \mathrm{mg} / \mathrm{dL}$; and platelet $=259,000 / \mathrm{mm}^{3}$, erythrocyte sedimentation rate $=29 \mathrm{~mm} / \mathrm{hrs}$; C-reactive protein $=$ negative. Laboratory tests for antinuclear antibodies (ANA) and anti-ds-DNA were provided to rule out lupus. The results of laboratory tests were positive for both ANA $(1: 2,560)$ and anti-ds-DNA antibodies. Cerebellar examination revealed abnormal finger-to-nose and tandem gait tests. Analysis of cerebrospinal fluid, brain magnetic resonance imaging (MRI), chest X-ray and abdominal-pelvic ultrasonography results were within normal limits. His electromyography (EMG) and nerve conductive velocity (NCV) study showed absent in compound sensory nerve action potentials. Therefore, he was diagnosed as having GBS according to Asbury criteria and admitted and received intravenous immunoglobulin (IVIG). However, there was no appropriate response by treatment and he developed severe headaches which were associated with disrupted sleep. Due to the presence of leukopenia during his admission, bone marrow aspiration was provided which was normal.

Considering polyneuropathy involving all limbs diagnosis was in favor of acute inflammatory demyelinating polyneuropathy. Cardiology consult was performed that showed mild mitral regurgitation and tricuspid regurgitation. Additional biochemical test analysis, as like as creatine phosphokinase; lactate dehydrogenase; thyroid function tests; Immunoglobulin levels, anticardiolipins antibodies; anti-smith antibodies; $\beta 2$ glycoproteins antibodies; and lupus anti-coagulant all were negative or within normal limits. Serological test results for brucellosis, mononucleosis, tuberculosis, bacterial and fungal and his throat culture were also negative. Random and $24 \mathrm{hrs}$ urinalysis test results were normal. The patient general condition was relatively improved during the hospitalization. Gait disorder was improved and the patient was discharged to be followed up.

Two weeks later, the patient was again admitted to our hospital with the complaint of legs pain, loss of balance, walking instability, lower extremity weakness, and anorexia. He had also ataxic gait. Cerebellar test disorders and DTR in lower limbs were observed as abnormal examinations. Distal force in upper and lower limbs was decreased. The patient was then admitted again in neurology ward. Lumbar puncture was performed again and auto-antibodies titration for GBS were checked which were in normal ranges. The serum level of vitamin B12 and vitamin E was measured to rule out the neuropathy that was normal. Total spinal MRI was normal. EMG and NCV tests were performed which reported chronic mild sensory motor demyelination in upper and lower limbs. The other biochemical and $\mathrm{CBC}$ tests results were performed again and showed normal results. The patient's general condition was gradually improved after supportive care and then be discharged.

However, he admitted again because of decreased force in upper and lower limbs (2/5), and absence of DTR in lower limbs due to the possibility of chronic inflammatory demyelinating polyneuropathy (CIDP). EMG and NCV tests showed chronic mild sensory motor demyelination in upper and lower limbs. Polyneuropathy involving all limbs suggestion was provided for CIDP. After CIDP diagnosis, the patient treated with the pulse of methylprednisolone for 5 days. He received oral prednisolone for 3 months and then gradually tapered. One month after stopping prednisone therapy, the patient developed severe upper and lower limbs weakness, walking instability, and gait disorders. The patient had decreased DTR in lower limbs under physical examinations. Finger-to-nose problems and tandem gait disorders were observed under cerebellar tests. Laboratory tests were performed again due to the possibility of lupus. The test results were as follow: anti GM IgM+, anti-CD10 IgG+, ANA+ (1:2,560), anti-ds-DNA+, and $\beta 2$ glycoprotein $\operatorname{IgM}+$. Proteinuria and hematuria were found under urinalysis. Leukopenia was found under several CBC tests analysis accompanied with low levels of complements. Eventually, the boy was transferred to division of Pediatric Rheumatology and treated with the pulse of methylprednisolone, hydroxychloroquine, and pulse of cyclophosphamide. His neurological and physical symptoms improved and had complete neurological recovery several months after his initial presentation. 


\section{Discussion}

SLE is an autoimmune systemic disease which is characterized by the production of autoantibodies against several organs; however, the mechanism is unknown. Due to the presence of autoantibodies, multiple body organs may be affected. For this reason, the clinical manifestations of SLE disease are complex and diverse. Common symptoms include joint pain and swelling, severe fatigue, loss of balance due to nerve involvement, headaches, rash, anemia, and blood-clotting disorders. Therefore, this disease may be misdiagnosed with symptoms of many other diseases. Neurological manifestations are common in patients with SLE. Nervous system involvement is frequently reported in $75 \%$ of patients in SLE. ${ }^{5}$ It is varied from a headache to acute confusional state, stroke, and myelopathy. ${ }^{6}$

GBS is a rare autoimmune disease in which autoantibodies preferentially target nerve cells in the peripheral nervous system. The exact cause of the disease is unclear; however, several factors such as viral or bacterial infection may be involved in GBS. ${ }^{3}$ Recent evidence has indicated that infection with Campylobacter may be the most common cause of GBS. ${ }^{7}$ Since autoantibodies attack peripheral nervous system in cases with GBS, nerves are damaged and unable to transmit signals from the brain to muscles. For this reason, GBS can be associated with multiple problems such as upper and lower limbs weakness, muscle weakness, bowel and bladder function problems, lower back pain, loss of balance, walking instability, numbness, and tingling sensation. Since these symptoms are very similar to those of other neurological disorders, the initial diagnosis of GBS is difficult. Lumbar puncture, electromyography, and nerve conduction tests are usually used for the diagnosis of GBS. ${ }^{8}$ Plasmapheresis or IVIG therapy are usually used for GBS treatment. ${ }^{8}$ Recent studies have reported several types of GBS. Acute inflammatory demyelinating polyradiculoneuropathy, Miller Fisher syndrome, acute motor axonal neuropathy, and acute motor-sensory axonal neuropathy are the most common forms of GBS. ${ }^{9}$

There may be an association between SLE and GBS; however, the exact mechanism is unknown. The incidence of SLE in patients with GBS has been estimated from $0.6 \%$ to $1.7 \% .{ }^{10}$ Until now, a few cases of SLE with initial presentation of GBS were reported. ${ }^{11}$ The first reported case was in 1964 with corticosteroid treatment alone and slow recovery or recurrences. The recent cases had treated with combination of IVIG, corticosteroid, intravenous cyclophosphamide, and/ or plasma exchange. Their outcome with this therapeutic protocol was complete or near complete resolution. For example, Nadri and Aithaf reported a 23-year-old female with initial presentation of GBS and final diagnosis of SLE. She was treated with intravenous pulse of methylprednisolone, IVIG, and plasmapheresis and a sustained clinical response was reported. ${ }^{11}$ Chandrashekhar and De Sousa reported an 18year-old woman suffered from SLE with initial presentation of GBS. Laboratory tests results for the patient revealed elevated ANA titer, positive antiphospholipid antibody panel, positive anti-ds-DNA, and low complement-4 (C4). She was treated with an intravenous pulse of methylprednisolone and plasmapheresis with remarkable improvement resulting in successful extubation and early ambulation. ${ }^{12}$ In another study, Fazio et al reported a 44-year-old woman with the diagnosis of GBS who suffered from progressive neurological disorders. Based on some laboratory test results such as elevated ANA titers, positive ds-DNA, and anti-Smith antibodies, SLE was eventually diagnosed. She was treated with IVIG but complicated with IVIG-induced splenic infarct. ${ }^{13}$ Similarly, Gao et al reported a 30-year-old female with numbness and lower extremities weakness who initially diagnosed with GBS. After further examinations with EMG and rheumatoidrelated results, SLE was diagnosed. Their patient had class V Lupus nephritis too. The patient was treated with intravenous pulses of methyl prednisolone for 5 days combined with pulse of cyclophosphamide monthly. Two months later, the patient's limb numbness and weakness disappeared. ${ }^{2}$ Vaidya et al previously reported a 23-year-old man with SLE who presented with GBS. Plasmapheresis, corticosteroids, IVIG and cyclophosphamide prescribed with good results in the patient. ${ }^{14}$

We reported a 12-year-old boy with juvenile SLE and initial presentation of GBS. Since GBS is variable in terms of the severity of involvement, it is important to diagnose the diseases in a timely manner to prevent potentially life-threatening complications. In our patient, suspicion for SLE was raised when the patient developed loss of balance, walking disability and lower-extremity weakness even after IVIG therapy due to the possibility of GBS. Furthermore, our case developed finger-to-nose abnormal test and tandem gait disorders under cerebellar tests. Our case had received oral prednisone for 3 months, but it was not efficient and the patient developed severe upper and lower limbs weakness, walking instability, and gait disorders. When GBS and SLE present concurrently, prednisone therapy is insufficient in about $50 \%$ of the cases. ${ }^{15}$ In this situations, cyclophosphamide 
along with pulse-dose steroids are usually used for SLE control. ${ }^{16}$ In our patient, according to American College of Rheumatology and Systemic Lupus International Collaborating Clinics Classification Criteria for SLE, the diagnosis of juvenile SLE was made with the presence of leukopenia, hemolytic anemia, hematuria, proteinuria, high level of ANA, decreased complement level, increased $\beta 2$ glycoprotein, and positive anti-ds-DNA antibody. The patient was treated with pulse methylprednisolone, intravenous cyclophosphamide, and hydroxychloroquine. His neurological and physical symptoms improved and had complete neurological recovery several months after his initial presentation.

\section{Conclusion}

Our case highlights that GBS can be an initial presentation of juvenile SLE. The GBS association with lupus is very rare which has likely an immunological basis. This association is important and must be recognized for rapid initiation of appropriate therapy. Furthermore, there are implications for both treatment and prognosis of the disease because symptoms of these diseases are almost similar to other neurological disorders. Our case presented with manifestations compatible with GBS but did not respond well to IVIG and prednisone as standard treatment. SLE was eventually diagnosed under further laboratory tests which showed the presence of leukopenia, hemolytic anemia, hematuria, proteinuria, positive ANA and ds-DNA antibody. He was treated with pulse of methylprednisolone, intravenous cyclophosphamide, and hydroxychloroquine. His neurological and physical signs and symptoms improved and had complete neurological recovery several months after his initial presentation.

\section{Consent}

We confirm that the written informed consent form has been provided by the parents to have the case details published. Also, we restate that institutional approval is not required to publish the case details.

\section{Acknowledgments}

This research did not receive any specific grant from funding agencies in the public, commercial, or not-forprofit sectors.

\section{Disclosure}

The authors report no conflicts of interest in this work.

\section{References}

1. Hsu TY, Wang SH, Kuo CF, Chiu TF, Chang YC. Acute inflammatory demyelinating polyneuropathy as the initial presentation of lupus. The American journal of emergency medicine. 2009 Sep 1;27(7):900-e3.

2. Gao Z, Li X, Peng T, et al. Systemic lupus erythematosus with GuillainBarre syndrome: a case report and literature review. Medicine (Baltimore). 2018;97:e11160. doi:10.1097/MD.0000000000011160

3. Okoh HC, Lubana SS, Langevin S, Sanelli-Russo S, Abrudescu A. A case of systemic lupus erythematosus presenting as Guillain-Barré syndrome. Case Rep Rheumatol. 2015;2015:1-5. doi:10.1155/2015/528026

4. Van Laarhoven HWM, Rooyer FA, van Engelen BGM, van Dalen R, Berden JHM. Guillain-Barré syndrome as presenting feature in a patient with lupus nephritis, with complete resolution after cyclophosphamide treatment. Nephrol Dial Transplant. 2001;16:840-842. doi:10.1093/ndt/16.4.840

5. Kakati S, Barman B, Ahmed SU, Hussain M. Neurological manifestations in systemic lupus erythematosus: a single centre study from North East India. J Clin Diagn Res. 2017;11:OC05-OC09. doi:10.7860/JCDR/2017/24731.9963

6. Appenzeller S, Costallat LT, Cendes F. Neurolupus. Arch Neurol. 2006;63:458-460. doi:10.1001/archneur.63.3.458

7. Van Doorn P. Diagnosis, treatment and prognosis of Guillain-Barré syndrome (GBS). La Presse Médicale. 2013;42:e193-e201. doi:10.1016/j.lpm.2013.02.328

8. Farcas P, Avnun L, Frisher S, Herishanu YO, Wirguin I. Efficacy of repeated intravenous immunoglobulin in severe unresponsive Guillain-Barré syndrome. Lancet. 1997;350:1747. doi:10.1016/ S0140-6736(97)24050-X

9. Kuwabara S. Guillain-Barré syndrome: epidemiology, pathophysiology and management. Drugs. 2004;64:597-610. doi:10.2165/ 00003495-200464060-00003

10. Korn-Lubetzki I, Abramsky O. Acute and chronic demyelinating inflammatory polyradiculoneuropathy. Association with autoimmune diseases and lymphocyte response to human neuritogenic protein. Arch Neurol. 1986;43:604-608.

11. Nadri Q, Althaf MM. Guillain-Barre syndrome as the initial presentation of systemic lupus erythematosus - case report and review of literature. Ann Saudi Med. 2015;35:263-265. doi:10.5144/02564947.2015.263

12. Chandrashekhar S, De Sousa E. Guillain-Barre syndrome as a rare initial presentation of systemic lupus erythematosus. Neurology. 2018;90(15 Supplement). doi:10.1212/WNL.0000000000005560

13. Fazio RM, Chen I, Somal N. Guillain-Barré syndrome as first presentation of systemic lupus erythematosus: a rare manifestation complicated by IVIg-induced splenic infarct. BMJ Case Rep. 2015;2015:211598.

14. Vaidya S, Jasin HE, Logan J. Systemic lupus erythematosus and Guillain-Barre syndrome. J Clin Rheumatol. 1999;5:349-353.

15. Chaudhuri KR, Taylor IK, Niven RM, Abbott RJ. A case of systemic lupus erythematosus presenting as Guillain-Barre syndrome. $\mathrm{Br}$ J Rheumatol. 1989;28:440-442.

16. Naveed A, Rampure R, Malik F, Jafari SIM, Amberker D. GuillainBarré syndrome occurring synchronously with systemic lupus erythematosus as initial manifestation treated successfully with low-dose cyclophosphamide. J Community Hosp Intern Med Perspect. 2016;6:30689 


\section{Publish your work in this journal}

Open Access Rheumatology Research and Reviews is an international, peer-reviewed, open access journal publishing original research, reports, editorials, reviews and commentaries on all aspects of clinical and experimental rheumatology in the clinic and laboratory including the following topics: Pathology, pathophysiology of rheumatological diseases; Investigation, treatment and management of rheumatological diseases; Clinical trials and novel pharmacological approaches for the treatment of rheumatological disorders. The manuscript management system is completely online and includes a very quick and fair peer-review system, which is all easy to use. Visit http://www.dovepress.com/testimonials.php to read real quotes from published authors. 